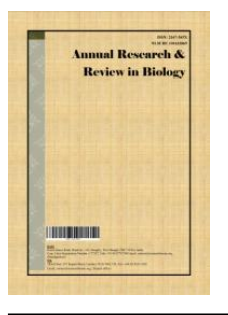

\title{
Neoplasias in Fish: Review of the Last 20 Years. A Look from the Pathology
}

\author{
Luis Alberto Romano ${ }^{1^{*}}$ and Virgínia Fonseca Pedrosa ${ }^{1}$ \\ ${ }^{1}$ Laboratory of Immunology and Pathology of Aquatic Organisms, Institute of Oceanography, Federal \\ University of Rio Grande, Rio Grande, Brazil.
}

Authors' contributions

This work was carried out in collaboration between both authors. The author LAR managed the writing and diagnoses reported in LIPOA and the author VFP collaborated with the literature search. Both authors read and approved the final manuscript.

Article Information

DOI: $10.9734 / A R R B / 2020 / v 35 i 1230319$ Editor(s):

(1) Dr. Viduranga Y. Waisundara, Australian College of Business \& Technology, Sri Lanka. Reviewers:

(1) Azhar M. Haleem, University of Technology, Iraq. (2) Shoaibe Hossain Talukder Shefat, Bangladesh. Complete Peer review History: http://www.sdiarticle4.com/review-history/64379

Review Article

Received 25 October 2020

Accepted 29 December 2020

Published 31 December 2020

\begin{abstract}
In fish there is an innumerable variety of neoplasias that arise essentially from all cell types. Neolasia here, we will focus on the neoplasias that appear spontaneously in these animals and will not cover the experimentally induced neoplasias and/or the animal models of neoplasias. As for diagnosis, in general, specialists in aquatic organism pathology are not so familiar with the diagnosis of neoplasias. Infectious pathology, as opposed to non-infectious pathology, is the predominant condition in this area and, of course, these are of greater importance because some infectious diseases generate great economic losses, while neoplasias are isolated pathologies, with some exceptions.

In the last 20 years, 10 neoplasias in different species have been diagnosed in our laboratory, and we reported their characteristics in this paper. We also made a detailed bibliography review and observed how 90 neoplasias among 56 species of teleosteal fish were reported.

Neoplasias in fish, unlike other diseases, do not generate great losses to aquaculture. However, the true value of neoplastic pathology compared is to better understand the histiogenesis and biological behavior of neoplasias in mammals and humans. Carcinogenesis is generally complex and in most neoplasias in both mammals and fish, the origin is unknown, and it seems that there are many factors that contribute to the onset and growth of neoplasias.
\end{abstract}


Keywords: Neoplasia; diagnosis; histopathology; immunohistochemistry; fish; carcinogenesis.

\section{INTRODUCTION}

Neoplasia literally means "new growth" and neoplasm is the result of this new growth. The term 'tumor' originally was applied to the swelling caused by the inflammation. Neoplasias can also induce swelling, but for a long time the term neoplastic has barely been used, so the term is now equivalent to neoplasia. Cancer, in turn, is the common and generic term for all malignant tumors, but in reality, it refers to carcinoma, a type of malignant neoplasia originating in epithelia. Although the ancient origins of this term are somewhat uncertain, it is probably derived from the Latin 'crab', cancer, presumably because a cancer "adheres to any part that stubbornly grabs like a crab" [1,2].

\section{NEOPLASMS IN TELEOST FISH}

In this review, we will adopt the term neoplasia in general and carcinoma and sarcoma in specific situations, with some exceptions, when unavoidable. Although all pathologists know what they mean when they use the term neoplasia, it has been surprisingly difficult to develop a precise definition. The eminent British oncologist Rupert Allan Willis [3] provided one of the most acceptable definitions: "A neoplasm is an abnormal mass of tissue, whose growth exceeds and is not coordinated with that of normal tissues and persists in the same excessive manner after the stimuli that triggered it have ceased". To this characterization we could add that the abnormal mass has no purpose, feeds on the host, and is virtually autonomous and, besides invading organs and interfering with their normal function and negatively impacting the immune system of a host, the neoplasias have their own histocompatibility antigens [4,5]. In fact, recent advances in cancer therapy in mammals and humans have shown clear benefits in stimulating the immune system. The inhibition of immune control points, whose efficacy requires that tumor antigens be recognized by $T$ cells, favors neoplasia growth. This critical step is mediated by interactions of the Major Histocompatibility Complex (MHC) with T-cell receptors. Class I $\mathrm{MHC}$ molecules are expressed by the majority of nucleated cells and contain mainly peptidic antigens derived endogenously from CD8 T cells [6].

When we say that neoplasias "feed on the host" it is because the neoplastic tissue competes with normal cells and tissues for supplying energy and the nourishing substrate for their growth. As these masses can multiply in an individual who is debilitated, they are to some extent autonomous. Further on, it becomes evident that this autonomy is not complete. All neoplasias ultimately depend on the host for their nutrition and vascular supply; many forms of neoplasias require endocrine support $[7,8]$. Some neoplastic cells are "immortal"; they die when the host dies, which indicates the intimate and intricate neoplasia/host relationship $[9,10]$.

The process of neoplasia is not limited to mammals; it can also develop in most vertebrates and in some insects and plants, and its occurrence in these other life forms has been useful to study its histiogenesis and its biological behavior [11-15].

In fish there is an innumerable variety of neoplasms that arise essentially from all cell types. In this review, we will focus on the neoplasms that appear spontaneously in these animals and we will not cover the experimentally induced neoplasias and/or the animal models of neoplasias, for which the fish has been of great scientific importance [16]. The frequency of neoplasias in fish is not negligible; only one author compiled 407 neoplasias in several species of fish and of various tissue origins a few decades ago [17]. On the other hand, several books on fish pathology dedicate some chapter to this theme [18-24].

All neoplasms, benign and malignant, have two basic components: first, neoplastic cells in proliferation that constitute their parenchyma and second, supporting stroma formed by connective tissue and blood vessels. Although the parenchymal cells represent the "vanguard" proliferation of neoplasias and, therefore, determine their nature; the growth and evolution of neoplasias critically depend on their stromatous environment [25]. An adequate stroma allows the supply of necessary blood to the stromatous connective tissue and provides a basis for the growth of neoplastic cells $[26,27]$. In some neoplasias, the stromatous support is scarce, causing it to be soft and fleshy. Sometimes parenchymal cells stimulate the formation of an abundant collagen stroma, called desmoplasia and therefore macroscopically are hard [28]. However, the nomenclature of 
neoplasms is based mainly on the parenchymatous component.

In general, benign neoplasias are designated by adding the suffix "oma" to the cell of origin. Mesenchymal cell neoplasias generally follow this rule. For example, a benign neoplasia that arises from fibroblastic cells is called fibroma [29], while a cartilaginous neoplasia is a chondroma and an osteoblast neoplasia is an osteoma [30-32]. In contrast, the nomenclature of benign epithelial neoplasms is more complex. They are classified in several ways, some based on their cells of origin, others on microscopic architecture and still others on their macroscopic patterns. A benign epithelial neoplasm arising from glandular cells would be called adenoma [33,34]. Benign epithelial neoplasias that produce projections in the form of fingers or warts, visible microscopically or macroscopically, from the epithelial surfaces, are called papillomas [35]. Those that form large cystic masses, as in the ovary, are called cystoadenomas [36]. Some tumors produce papillary patterns that stand out through the cystic spaces and are called papillary cystoadenomas [37].

The nomenclature of malignant neoplasms follows essentially the same scheme used for benign ones, with some additions. Those arising from mesenchymal tissue are usually called sarcomas, such as fibrosarcoma, liposarcoma and leiomyosarcoma for smooth muscle neoplasms, and rhabdomyosarcoma for striated muscle neoplasms [38-41]. Epithelial cell derivatives are called carcinomas. Thus, the neoplasia that appears in the epidermis of ectodermal origin is a carcinoma, similar to the neoplasias that appear in the mesodermically derived cells of the renal tubules and endodermically derived cells of the lining of the gastrointestinal tract [42]. Carcinomas can also be qualified by their pattern and architecture; the glandular growth microscopically identified is called adenocarcinoma, and the one that produces recognizable squamous cells, which appears in any epithelium of the body, is called squamous cell carcinoma or epidermoid carcinoma. It is an additional common practice to specify, the source organ where possible [34]. However, it is not uncommon for a neoplasia to consist of undifferentiated cells and must be designated simply as a malignant neoplasia with little or no differentiation $[43,44]$.
In most neoplasias, parenchymal cells look very much like each other, as if they were all derived from a single cell. Less frequently, the divergent differentiation of a single lineage of parenchymal cells creates what are called mixed neoplasias. The best example is the so-called carcinosarcomas; these tumors contain scattered epithelial components within a sarcomatous stroma which sometimes contain apparent cartilage islands or even bone [45]. The vast majority of neoplasias, including mixed neoplasias, are composed of cells representing a single embryonic layer. The teratoma, by contrast, is formed by a variety of parenchymal cell types representative of more than one germ layer, generally all three. They arise from totipotential cells and, therefore, are found mainly in the gonads, even if, on rare occasions, they are found in remnants of primitive cells abducted elsewhere. These totipotential cells differentiate themselves along several germinal pathways, producing, for example, tissues that can be identified as skin, muscle, fat, dental structures and, in fact, any body tissue [46-48].

The nomenclature of neoplasias is important because specific designations have specific biological implications. In the most common forms of neoplasia their name is related to histiogenesis and its biological behavior (benign or malignant). However, there are some inappropriate but deeply rooted uses. For generations, melanocyte neoplasias have been called melanomas, although they should be correctly called melanocarcinomas [49]. Similarly, lymphomas that are malignant, from different biological behaviors, are called with the suffix "oma", which would seem to imply benignity [50].

Similarly, aberrant differentiation can produce a mass of disorganized but mature specialized cells where the particular site is known as hamartoma. Therefore, a hamartoma may contain small islands of cartilage, blood vessels and lymphoid tissue. Sometimes the lesion is purely cartilaginous or purely angiomatous. Even if they can be interpreted as benign neoplasias, the total similarity of the tissue to cartilage or normal blood vessels and the occasional mixing of other elements favors a hamartomatous origin. In any case, hamartoma is totally benign $[51,52]$.

In the vast majority of cases, the differentiation between a benign and a malignant neoplasia can be made morphologically with considerable 
accuracy; sometimes, however, a neoplasia challenges categorization. It has already been said: "it is not necessary that all neoplasias be classified as benign or malignant". Certain histological characteristics may suggest innocence, while others point to an aggressive potential. Ultimately, every morphological diagnosis is subjective and constitutes a prediction of the future course of a neoplasia [53]. In some cases, this prediction is confused by a marked discrepancy between the morphological appearance of a neoplasia and its biological behavior. However, such deception or ambiguity is not the rule; in general, there are criteria by which benign neoplasias can be distinguished from malignant ones, which will behave according to such definition. These differences are related to differentiation and anaplasia of the cells that compose the neoplasia, their growth rate, invasion capacity, and ability to produce metastasis [54].

The terms 'differentiation' and 'anaplasia' apply to the parenchymal cells of neoplasia. Differentiation refers to the degree to which parenchymal cells resemble normal comparative cells, both morphologically and functionally. Therefore, well differentiated neoplasias are composed of cells that resemble the normal mature cells of the tissue of origin of the neoplasia. Poorly differentiatedor undifferentiated neoplasms have unspecialized cells of primitive appearance. In general, benign neoplasias are well differentiated. Smooth muscle neoplasia, a leiomyoma, resembles so much the normal cell that it is impossible to recognize it as a tumor cell in a high-resolution examination. Only by the massification of these cells, by counting and cell differentiation in a nodule, the neoplastic nature of the lesion is revealed, and it is possible to get closer to the 'tree that has lost sight of the forest' [55].

Malignant neoplasias, in turn, vary from well differentiated to undifferentiated. It is said that malignant neoplasias composed of undifferentiated cells are anaplastic. The lack of differentiation, or anaplasia, is considered a distinctive seal of malignant transformation. Literally, anaplasia means "reverse formation", which implies a reversion from a high level of differentiation to a lower level. However, there is substantial evidence that neoplasias arise from mother cells present in all specialized tissues. A well differentiated neoplasia evolves from a maturation or specialization of undifferentiated cells as they proliferate, while an undifferentiated neoplasia evolves without maturation of the transformed cells [56]. Thus, the lack of differentiation is not a consequence of 'dedifferentiation'. The lack of differentiation, or anaplasia, is marked by a series of morphological and functional changes. In a characteristic way, both the cells and the nuclei present a variation of pleomorphism in size and shape. It is possible to find cells that are many times larger than their neighbors and others that can be extremely small and primitive looking, and this is called anisocytosis. Characteristically, the nuclei of these cells contain a great amount of DNA and have a very dark coloration (hypercromatic). They are disproportionately large in relation to the cell and the proportion of the nucleus to the cytoplasm may reach $1: 1$, instead of the normal proportion of $1: 4$ or $1: 6$. The shape of the nucleus can be extremely variable, and the chromatin is usually grouped and roughly distributed along the nuclear membrane and this is called anisocytosis. Large nucleoli are usually present in these nuclei [57].

Compared to benign neoplasias and some well differentiated malignancies, undifferentiated ones generally have many mitoses, which reflects the greater proliferating activity of parenchymal cells [58]. The presence of mitosis, however, does not necessarily indicate malignancy or even that it is neoplastic tissue. Many normal tissues that exhibit a rapid change, such as the lymphohematopoietic cells of the pronefros, have numerous mitoses and the non-neoplastic proliferations, such as hyperplasia, contain many cells in the mitosis process [59]. More important as morphological characteristics of malignant neoplasia are the strange and atypical mitotic figures that sometimes produce tripolar, quadripolar or multipolar spindles. Another characteristic of anaplasia is the formation of giant tumor cells, where some have only one huge polymorphic nucleus and others have two or more nuclei. These giant cells should not be confused with those that are inflammatory, such as the enormous cells present in a foreign body or the typical Langhans cells of mycobacteria. In the neoplastic giant cell, the nucleus is hypercromatic and is large in relation to the cell [60]. Besides the cytological anomalies described here, the orientation of the anaplastic cells is markedly altered, that is, they lose their normal polarity, observing large masses of neoplastic cells that grow anarchically and in a disorganized manner [61]. Although these growing cells obviously require a blood supply, often the vascular stroma is scarce, and in many 
anaplastic neoplasias, large central areas suffer ischemic necrosis [62].

It may be generalized that most benign neoplasms grow slowly over a period of years, while most malignancies grow rapidly, sometimes at an erratic rate, and eventually can spread and kill their hosts. At the other extreme are the neoplasms that grow more slowly and can also enter periods of lethargy that last for years.

A characteristic of many benign neoplasias is the development of a compression edge of connective tissue, sometimes called a fibrous capsule, which separates them from the host tissue. This capsule is largely derived from the stroma of native tissue as the parenchymal cells atrophy under the pressure of the expanding neoplasia. Such an encapsulation process tends to contain benign neoplasia as a discrete mass. Although there is this well-defined excision plane around most benign neoplasias, in some cases this process is not present. Therefore, hemangiomas (neoplasms composed of entangled blood vessels) are often not encapsulated and may appear to impregnate the site where they appear [63]. The growth of malignant neoplasias is accompanied by infiltration, invasion, and progressive destruction of surrounding tissue. The histological examination of these apparently encapsulated masses almost always reveals small neoplastic growth that penetrates the margin and infiltrates the adjacent structures [64].

Most malignant neoplasias are obviously invasive and can be expected to penetrate the surrounding tissue. Along with the development of metastasis, the degree of invasion is the most reliable feature that differentiates malignant from benign neoplasias.

Metastases are implants of discontinuous neoplastic tissue with the primary tumor. Metastasis unequivocally marks a neoplasia as malignant because benign ones do not cause metastasis [65].

It is therefore evident that the properties of invasion and metastasis are separable. In general, the more aggressive, faster growing, and greater the primary neoplasia, the greater is the probability that it already has metastasis. However, there are countless exceptions. Small, well differentiated, and slow growing lesions sometimes have a wide metastasis and, on the contrary, some fast-growing lesions may remain localized for years. The probability of metastasis cannot be judged from the pathological examination of primary neoplasia [66-68].

As for diagnosis, experts in aquatic organism pathology are generally not so familiar with diagnosing neoplasias. Infectious pathology, as opposed to non-infectious pathology, is the predominant one in this area and, clearly, these are of greater importance because some infectious diseases generate great economic losses, while neoplasms are isolated pathologies, with some exceptions, such as the case of melanomas in Xiphophorus, which have a genetic implication that can alter the production of these ornamental fish in aquaculture $[69,70]$.

Although histopathology is a very useful tool for the diagnosis of diseases in general, in the case of neoplasias it has a fundamental role in diagnosis [71]. First, for the differential diagnosis, when observing the tissues, the first question is whether it is an inflammatory or neoplastic pathology. With some exceptions, such as certain inflammatory pseudotumors, the histopathological difference is clear, presenting no problem in differentiating between these pathologies [72]. When it is determined that it is a neoplasia, two questions immediately arise about its histogenesis and its biological behavior: from which tissue did this neoplasia originate? is it a benign or malignant neoplasia? In a high percentage of cases, with optical microscopy with $\mathrm{H}-\mathrm{E}$ and other complementary techniques with dyes, the question is resolved [73]. In other cases, where the neoplasias are little differentiated or undifferentiated, the problem is aggravated. In our laboratory, in the last 20 years, 10 neoplasias were diagnosed in different species, whose characteristics can be seen in Table 1.

We performed a detailed bibliography review and observed how 90 neoplasias were reported among 56 species of teleosteal fish (Table 2-13).

Coinciding with Stoskopf [21], we observed that cyprinids are the ones that present more neoplasias. The author associates this to the fact that they have a very long life.

The vertiginous development of diagnostic methods and techniques in human medicine was immediately transferred to veterinary medicine and therefore to the pathology of aquatic organisms, which is a branch of veterinary medicine. 
Table 1. Spontaneous neoplasms in teleost fish related in LIPOA between 2000-2020

\begin{tabular}{|c|c|c|c|c|c|c|}
\hline \multirow[t]{2}{*}{ Neoplasm } & \multirow[t]{2}{*}{ Species } & \multirow[t]{2}{*}{ Year } & \multicolumn{3}{|c|}{ Diagnosis } & \multirow[t]{2}{*}{ References } \\
\hline & & & OM & IHQ & EM & \\
\hline Thymoma & Cyprinus carpio & 2004 & Yes & No & Yes & Romano and Marozzi, 2004 [74] \\
\hline Carcinosarcoma of mandible & Onchorhyincus mykiss & 2010 & Yes & Yes & No & Romano et al., 2010 [45] \\
\hline Neoplasia of the sertoli cells & Cyprinus carpio & 2013 & Yes & Yes & Yes & Romano et al., 2013 [75] \\
\hline Borderline ovarian cystadenoma & Carasius auratus & 2014 & Yes & Yes & No & Romano et al., 2014[ 64] \\
\hline Multiple neurofibromas of the heart & Cyprinus carpio & 2015 & Yes & Yes & Yes & Romano et al., 2015 [76] \\
\hline Alveolar rhabdomyosarcoma & Carasius auratus & 2016 & Yes & Yes & Yes & Wicki et al., 2016 [41] \\
\hline Melanoma & Carasius auratus & 2018 & Yes & Yes & Yes & Romano et al., 2018 [77] \\
\hline Haemangioma esclerosante & Paralichthys orbignyanus & 2018 & Yes & No & No & Klosterhoff et al., 2018[ 63] \\
\hline Lymphoma & Rachycentron canadum & 2019 & Yes & Yes & No & Klosterhoff et al., 2019 [50] \\
\hline Melanoma & Xiphophorus hellerii & 2020 & Yes & Yes & Yes & Romano et al., 2020 [78] \\
\hline
\end{tabular}

*OM: Optical Microscopy; IHQ: Immunohistochemistry; EM: Electron Microscopy

Table 2. Spontaneous neoplasms in teleost fish related between 2000-2020

\begin{tabular}{|c|c|c|c|c|c|c|c|}
\hline \multirow[t]{2}{*}{ Family } & \multirow[b]{2}{*}{ Neoplasm } & \multirow[b]{2}{*}{ Species } & \multirow[b]{2}{*}{ Year } & \multicolumn{3}{|c|}{ Diagnosis } & \multirow[b]{2}{*}{ References } \\
\hline & & & & OM & IHQ & EM & \\
\hline \multirow[t]{2}{*}{ Acipenseridae } & Haemangioma & Acipenser baerii & 2018 & Yes & No & No & Rezaie et al. 2018 [79] \\
\hline & Nephroblastoma & Huso huso x Acipenser ruthenus & 2018 & Yes & Yes & No & Rahmati-Holasoo et al. 2018 [80] \\
\hline Adrianichthyidae & Lymphoma & Oryzias latipes & 2008 & Yes & No & No & Hayashi et al. 2008 [81] \\
\hline \multirow[t]{2}{*}{ Anguillidae } & Stomatopapillomatosis & Anguilla anguilla & 2010 & Yes & Yes & No & Marino et al. $2010^{a}[82]$ \\
\hline & Myxoma & Anguilla anguilla & 2014 & Yes & Yes & No & Gjurcevic et al., 2014 [83] \\
\hline Ariidae & Fibrosarcoma & Hemiarius dioctes & 2015 & Yes & No & No & Dennis and Diggles, 2015 [40] \\
\hline Callichthyidae & Skin nodular neoplasm & Callichthys callichthys & 2003 & Yes & No & No & Caruso et al. 2003 [84] \\
\hline Characidae & Cutaneous ossifying fibroma & Paracheirodon innesi & 2016 & Yes & No & No & Murphy and Imai, 2016 [29] \\
\hline
\end{tabular}

from those used by the authors mentioned in the table. OM: Optical Microscopy; IHQ: Immunohistochemistry; EM: Electron Microscopy 
Table 3. Spontaneous neoplasms in teleost fish related between 2000-2020

\begin{tabular}{|c|c|c|c|c|c|c|c|}
\hline \multirow[t]{2}{*}{ Family } & \multirow[b]{2}{*}{ Neoplasm } & \multirow[b]{2}{*}{ Species } & \multirow[b]{2}{*}{ Year } & \multicolumn{3}{|c|}{ Diagnosis } & \multirow[b]{2}{*}{ References } \\
\hline & & & & MO & IHQ & ME & \\
\hline \multirow[t]{4}{*}{ Cichlidae } & Lymphoma & Hua Luo-Han & 2008 & Yes & No & No & Lin et al. 2008 [85] \\
\hline & Adenoma & Astronotus ocellatus & 2010 & Yes & No & Yes & Hochwartner et al. 2010 [86] \\
\hline & Squamous cell carcinoma & Astronotus ocellatus & 2010 & Yes & No & No & Rahmati-holasoo et al. 2010 [87] \\
\hline & Liposarcoma & Flower horn fish & 2016 & Yes & No & No & Rahmati-Holasoo et al. 2016 [88] \\
\hline Clariidae & Rhabdomyosarcoma & Clarias gariepinus & 2017 & Yes & No & No & Okorie-Kanu et al. 2017 [89] \\
\hline Clinidae & Chromatophoroma & Gibbonsia montereyensis & 2011 & Yes & Yes & Yes & Camus et al. $2011[90]$ \\
\hline Clupeidae & Leiomyoma & Sardina pilchardus & 2003 & Yes & No & No & Ramos and Peleteiro, 2003 [91] \\
\hline Congridae & Angioleiomyoma & Conger conger & 2016 & Yes & Yes & No & Marino et al. 2016 [92] \\
\hline
\end{tabular}

Table 4. Spontaneous neoplasms in teleost fish related between 2000-2020

\begin{tabular}{|c|c|c|c|c|c|c|c|}
\hline \multirow[t]{2}{*}{ Family } & \multirow[b]{2}{*}{ Neoplasm } & \multirow[b]{2}{*}{ Species } & \multirow[b]{2}{*}{ Year } & \multicolumn{3}{|c|}{ Diagnosis } & \multirow[b]{2}{*}{ References } \\
\hline & & & & MO & IHQ & ME & \\
\hline Cottidae & Squamous cell carcinoma & Cottus gobio & 2016 & Yes & Yes & No & Quaglio et al. 2016[93] \\
\hline \multirow[t]{7}{*}{ Cyprinidae } & Melanoma & Cyprinus japonicus & 2003 & Yes & No & No & Ramos and Peleteiro, 2003[91] \\
\hline & Haemangioma & Carassius auratus & 2006 & Yes & No & No & O'Hagan and Raidal, 2006[94] \\
\hline & Ovarian carcinoma & Cyprinus carpio & 2006 & Yes & Yes & No & Raidal et al. 2006[95] \\
\hline & Branchioblastoma & Cyprinus carpio & 2007 & Yes & No & No & Knüsel et al. 2007[96] \\
\hline & Branchioblastoma & Cyprinus carpio & 2010 & Yes & No & No & Sholichah et al. 2010[97] \\
\hline & Nephroblastoma & Cyprinus carpio & 2010 & Yes & No & No & Stegeman et al. 2010[98] \\
\hline & Haemangioma & Puntius sarana & 2012 & Yes & No & No & Sahoo et al. 2012[99] \\
\hline
\end{tabular}

*The names of the neoplasms included in table 2 are those used by the authors cited in the references. The terminology of neoplasms diagnosed in our laboratory often differs from those used by the authors mentioned in the table. OM: Optical Microscopy; IHQ: Immunohistochemistry; EM: Electron Microscopy 
Table 5. Spontaneous neoplasms in teleost fish related between 2000-2020

\begin{tabular}{|c|c|c|c|c|c|c|c|}
\hline \multicolumn{8}{|l|}{ Family } \\
\hline & \multirow[b]{2}{*}{ Neoplasm } & \multirow[b]{2}{*}{ Species } & \multirow[b]{2}{*}{ Year } & \multicolumn{3}{|c|}{ Diagnosis } & \multirow[b]{2}{*}{ References } \\
\hline & & & & MO & IHQ & ME & \\
\hline \multirow[t]{8}{*}{ Cyprinidae } & Schwannoma & Danio rerio & 2012 & Yes & Yes & No & Marino et al. 2012[100] \\
\hline & Haemangiosarcoma & Cyprinus carpio & 2013 & Yes & No & No & Hyatt et al. 2013[101] \\
\hline & Ocular glioneuroma & Carassius auratus & 2014 & Yes & Yes & No & Mandrioli et al. 2014[102] \\
\hline & Leiomyoma & Carassius auratus & 2015 & Yes & Yes & No & Oryan et al. 2015[103] \\
\hline & Intestinal carcinoma & Danio rerio & 2015 & Yes & Yes & No & Paquette et al. 2015[104] \\
\hline & Schwannoma & Carassius auratus & 2015 & Yes & Yes & Yes & Sirri et al. 2015a[57] \\
\hline & Papilloma & Carassius auratus & 2016 & Yes & Yes & No & Lanteri et al. 2016[105] \\
\hline & Chondroma & Ctenopharyngodon idella & 2016 & Yes & No & No & Mesbah et al. 2016[31] \\
\hline
\end{tabular}
from those used by the authors mentioned in the table. OM: Optical Microscopy; IHQ: Immunohistochemistry; EM: Electron Microscopy

Table 6. Spontaneous neoplasms in teleost fish related between 2000-2020

\begin{tabular}{|c|c|c|c|c|c|c|c|}
\hline \multicolumn{8}{|l|}{ Family } \\
\hline & \multirow[b]{2}{*}{ Neoplasm } & \multirow[b]{2}{*}{ Species } & \multirow[b]{2}{*}{ Year } & \multicolumn{3}{|c|}{ Diagnosis } & \multirow[b]{2}{*}{ References } \\
\hline & & & & MO & IHQ & ME & \\
\hline \multirow[t]{8}{*}{ Cyprinidae } & Rhabdomyosarcoma & Hypophthalmichthys molitrix & 2016 & Yes & No & No & Rezaie et al. 2016[106] \\
\hline & Fibroma & Carassius auratus & 2016 & Yes & No & No & Shokrpoor et al. 2016[107] \\
\hline & Myxoma & Carassius auratus & 2016 & Yes & No & No & Shokrpoor et al. 2016[107] \\
\hline & Haemangiopericytoma & Cyprinus carpio & 2016 & Yes & Yes & Yes & Sirri et al. 2016[108] \\
\hline & Fibrosarcoma & Carassius auratus & 2017 & Yes & No & No & Rezaie et al. 2017a[109] \\
\hline & Chromatophoroma & Cyprinus carpio & 2019 & Yes & Yes & No & Siniard et al. 2019[110] \\
\hline & Chromatophoroma & Carassius auratus & 2019 & Yes & Yes & No & Siniard et al. $2019[110]$ \\
\hline & Papilloma & Abramis brama & 2019 & Yes & Yes & No & laria et al. 2019[111] \\
\hline
\end{tabular}

${ }^{*}$ The names of the neoplasms included in table 2 are those used by the authors cited in the references. The terminology of neoplasms diagnosed in our laboratory often differs from those used by the authors mentioned in the table. OM: Optical Microscopy; IHQ: Immunohistochemistry; EM: Electron Microscopy 
Table 7. Spontaneous neoplasms in teleost fish related between 2000-2020

\begin{tabular}{|c|c|c|c|c|c|c|c|}
\hline \multicolumn{8}{|l|}{ Family } \\
\hline & \multirow[b]{2}{*}{ Neoplasm } & \multirow[b]{2}{*}{ Species } & \multirow[b]{2}{*}{ Year } & \multicolumn{3}{|c|}{ Diagnosis } & \multirow[b]{2}{*}{ References } \\
\hline & & & & OM & IHQ & EM & \\
\hline Cyprinidae & Schwannoma & Carassius carassius & 2019 & Yes & Yes & No & laria et al. 2019[111] \\
\hline Goodeidae & Ovarian teratoma & Zoogoneticus tequila & 2016 & Yes & Yes & No & Romanucci et al. 2016[48] \\
\hline Lepisosteidae & Ovarian cystadenoma & Lepisosteus oculatus & 2000 & Yes & No & No & Thiyagarajah et al. 2000[36] \\
\hline Lutjanidae & Neurofibroma & Lutjanus griseus & 2000 & Yes & No & No & Williams et al. 2000[112] \\
\hline \multirow[t]{2}{*}{ Moronidae } & Fibropapiloma & Dicentrarchus labrax & 2003 & Yes & No & No & Ramos and Peleteiro, 2003[91] \\
\hline & Lipoma & Dicentrarchus labrax & 2011 & Yes & No & No & Marino et al. 2011[113] \\
\hline Mugilidae & Neurofibroma & Mugil cephalus & 2010 & Yes & Yes & No & Marino et al. 2010b[114] \\
\hline Osphronemidae & Rhabdomyosarcoma & Colisa fasciata & 2001 & Yes & No & No & Iwanowicz et al. 2001[115] \\
\hline
\end{tabular}

Table 8. Spontaneous neoplasms in teleost fish related between 2000-2020

\begin{tabular}{|c|c|c|c|c|c|c|c|}
\hline \multicolumn{8}{|l|}{ Family } \\
\hline & \multirow[b]{2}{*}{ Neoplasm } & \multirow[b]{2}{*}{ Species } & \multirow[b]{2}{*}{ Year } & \multicolumn{3}{|c|}{ Diagnosis } & \multirow[b]{2}{*}{ References } \\
\hline & & & & OM & IHQ & EM & \\
\hline \multirow[t]{3}{*}{ Osphronemidae } & Nephroblastoma & Betta splendens & 2010 & Yes & No & No & Lombardini et al. 2010[116] \\
\hline & Melanoma & Betta splendens & 2014 & Yes & Yes & No & Rahmati-Holasoo et al. 2015[117] \\
\hline & Iridophoroma & Betta splendens & 2019 & Yes & No & No & Rahmati-Holasoo et al. 2019[118] \\
\hline Ostraciidae & Iridociliary melanoma & Lactoria cornuta & 2010 & Yes & No & No & Da Silva et al. 2010[119] \\
\hline Percidae & Fibrosarcoma & Perca flavescens & 2005 & Yes & No & No & Bowser et al. 2005[120] \\
\hline \multirow[t]{3}{*}{ Poeciliidae } & Melanoma & Xiphophorus nezahualcoyotl & 2008 & Yes & No & No & Fernandez and Bowser, 2008[70] \\
\hline & Lipoma & Poecilia velifera & 2012 & Yes & No & No & De Stefano et al. 2012[121] \\
\hline & Congenital teratoma & Poecilia reticulata & 2014 & Yes & No & No & Rahmati-holasoo et al. $2014[122]$ \\
\hline
\end{tabular}

${ }^{*}$ The names of the neoplasms included in table 2 are those used by the authors cited in the references. The terminology of neoplasms diagnosed in our laboratory often differs from those used by the authors mentioned in the table. OM: Optical Microscopy; IHQ: Immunohistochemistry; EM: Electron Microscopy 
Table 9. Spontaneous neoplasms in teleost fish related between 2000-2020

\begin{tabular}{|c|c|c|c|c|c|c|c|}
\hline \multicolumn{8}{|l|}{ Family } \\
\hline & Neoplasm & Species & Year & OM & $\mathrm{IHQ}$ & EM & References \\
\hline \multirow[t]{2}{*}{ Poeciliidae } & Squamous cell carcinoma & Xiphophorus maculatus & 2017 & Yes & No & No & Rezaie et al. 2017b[123] \\
\hline & Melanoma & Xiphophorus sp. & 2019 & Yes & Yes & No & laria et al. 2019[111] \\
\hline Pomacentridae & Odontoma & Amphiprion ocellaris & 2018 & Yes & No & No & Vorbach et al. 2018[52] \\
\hline \multirow[t]{5}{*}{ Salmonidae } & Renal carcinoma & Oncorhynchus tshawytscha & 2003 & Yes & No & No & Lumsden and Marshall, 2003[124] \\
\hline & Renal cystadenoma & Oncorhynchus tshawytscha & 2003 & Yes & No & No & Lumsden and Marshall, 2003[124] \\
\hline & Biliary cystadenoma & Oncorhynchus tshawytscha & 2003 & Yes & No & No & Lumsden and Marshall, 2003[124] \\
\hline & Leiomyoma & Oncorhynchus tshawytscha & 2003 & Yes & No & No & Lumsden and Marshall, 2003[124] \\
\hline & Rhabdomyosarcoma & Oncorhynchus tshawytscha & 2003 & Yes & No & No & Lumsden and Marshall, 2003[124] \\
\hline
\end{tabular}

differs from those used by the authors mentioned in the table. OM: Optical Microscopy; IHQ: Immunohistochemistry; EM: Electron Microscopy

Table 10. Spontaneous neoplasms in teleost fish related between $2000-2020$

\begin{tabular}{|c|c|c|c|c|c|c|c|}
\hline \multicolumn{8}{|l|}{ Family } \\
\hline & \multirow[b]{2}{*}{ Neoplasm } & \multirow[b]{2}{*}{ Species } & \multirow[b]{2}{*}{ Year } & \multicolumn{3}{|c|}{ Diagnosis } & \multirow[b]{2}{*}{ References } \\
\hline & & & & OM & IHQ & EM & \\
\hline \multirow[t]{3}{*}{ Salmonidae } & Peri-hepatic teratoma & Oncorhynchus tshawytscha & 2003 & Yes & No & No & Lumsden and Marshall, 2003[124] \\
\hline & Leiomyosarcoma & Salmo salar & 2012 & Yes & Yes & No & Bowser et al. 2012[38] \\
\hline & Rhabdomyosarcoma & Salvelinus fontinalis & 2015 & Yes & Yes & Yes & Sirri et al. 2015b[125] \\
\hline Sciaenidae & Sarcoma thymic & Argyrosomus regius & 2012 & Yes & No & Yes & Soares et al. 2012[126] \\
\hline \multirow[t]{3}{*}{ Scombridae } & Lipoma & Thunnus thynnus & 2006 & Yes & No & No & Marino et al. 2006[127] \\
\hline & Lipoma & Thunnus maccoyii & 2008 & Yes & Yes & No & Johnston et al. 2008[128] \\
\hline & Neurofibrosarcoma & Thunnus maccoyii & 2008 & Yes & Yes & No & Johnston et al. 2008[128] \\
\hline Serranidae & Seminoma & Centropristis striata & 2002 & Yes & No & No & Weisse et al. 2002[129] \\
\hline
\end{tabular}

*The names of the neoplasms included in table 2 are those used by the authors cited in the references. The terminology of neoplasms diagnosed in our laboratory often differs from those used by the authors mentioned in the table. OM: Optical Microscopy; IHQ: Immunohistochemistry; EM: Electron Microscopy 


\section{Table 11. Spontaneous neoplasms in teleost fish related between 2000-2020}

\begin{tabular}{|c|c|c|c|c|c|c|c|}
\hline \multicolumn{8}{|l|}{ Family } \\
\hline & Neoplasm & Species & Year & OM & $I H Q$ & EM & References \\
\hline \multirow[t]{3}{*}{ Sparidae } & Lymphoma & Pagrus major & 2000 & Yes & No & Yes & Miyazaki et al. 2000[65] \\
\hline & Melanoma & Plectropomus leopardus & 2012 & Yes & No & Yes & Sweet et al. 2012[130] \\
\hline & Schwannoma & Sparus aurata & 2008 & Yes & Yes & No & Marino et al. $2008[131]$ \\
\hline Sparidae & Lipoma & Lithognathus mormyrus & 2009 & Yes & No & No & Gómez, 2009[132] \\
\hline \multirow[t]{4}{*}{ Sparidae } & Lymphoma & Rhabdosargus sarba & 2017 & Yes & No & Yes & Singaravel et al. 2017[133] \\
\hline & Iridophoric osteoma & Rhabdosargus sarba & 2017 & Yes & No & Yes & Singaravel et al. 2017[133] \\
\hline & Osteoma & Rhabdosargus sarba & 2017 & Yes & No & Yes & Singaravel et al. 2017[133] \\
\hline & Psammomatoid Ossifying fibroma & Rhabdosargus sarba & 2017 & Yes & No & Yes & Singaravel et al. 2017[133] \\
\hline
\end{tabular}

differs from those used by the authors mentioned in the table. OM: Optical Microscopy; IHQ: Immunohistochemistry; EM: Electron Microscopy

\section{Table 12. Spontaneous neoplasms in teleost fish related between 2000-2020}

\begin{tabular}{|c|c|c|c|c|c|c|c|}
\hline \multicolumn{8}{|l|}{ Family } \\
\hline & \multirow[b]{2}{*}{ Neoplasm } & \multirow[b]{2}{*}{ Species } & \multirow[b]{2}{*}{ Year } & \multicolumn{3}{|c|}{ Diagnosis } & \multirow[b]{2}{*}{ References } \\
\hline & & & & OM & IHQ & EM & \\
\hline Sparidae & Fibroma & Lithognathus mormyrus & 2019 & Yes & Yes & No & laria et al. 2019 [111] \\
\hline Sphyraenidae & Leiomyosarcoma & Sphyraena jello & 2017 & Yes & No & No & Singaravel et al. 2017 [133] \\
\hline \multirow[t]{6}{*}{ Syngnathidae } & Fibrosarcoma & Hippocampus erectus & 2004 & Yes & No & No & Willens et al. 2004 [134] \\
\hline & Renal adenoma & Hippocampus kuda & 2012 & Yes & No & No & LePage et al. 2012 [135] \\
\hline & Renal adenocarcinoma & Hippocampus kuda & 2012 & Yes & No & No & LePage et al. 2012 [135] \\
\hline & $\begin{array}{l}\text { Exocrine pancreatic } \\
\text { carcinoma }\end{array}$ & Hippocampus kuda & 2012 & Yes & No & No & LePage et al. 2012 [135] \\
\hline & Intestinal carcinoma & Hippocampus kuda & 2012 & Yes & No & & LePage et al. 2012 [135] \\
\hline & Rhabdomyosarcoma cardiac & Phyllopteryx taeniolatus & 2012 & Yes & No No & & LePage et al. 2012 [135] \\
\hline
\end{tabular}

*The names of the neoplasms included in table 2 are those used by the authors cited in the references. The terminology of neoplasms diagnosed in our laboratory often differs from those used by the authors mentioned in the table. OM: Optical Microscopy; IHQ: Immunohistochemistry; EM: Electron Microscopy 
Table 13. Spontaneous neoplasms in teleost fish related between $\mathbf{2 0 0 0 - 2 0 2 0}$

\begin{tabular}{|c|c|c|c|c|c|c|c|}
\hline \multirow[t]{2}{*}{ Family } & \multirow[b]{2}{*}{ Neoplasm } & \multirow[b]{2}{*}{ Species } & \multicolumn{4}{|c|}{ Diagnosis } & \multirow[b]{2}{*}{ References } \\
\hline & & & Year & OM & IHQ & EM & \\
\hline Syngnathidae & Adenoma & Hippocampus erectus & 2018 & Yes & Yes & No & Stilwell et al. 2018 [136] \\
\hline Trichiuridae & Osteoma & Trichiurus lepturus & 2002 & Yes & No & No & Lima et al. 2002 [137] \\
\hline
\end{tabular}

In the 1970s, electronic microscopy clearly collaborated in the precise diagnosis of neoplasias [138]. This technique has proved to be essential for the diagnosis of certain diseases; however, its application to the diagnosis of neoplasias in general has been relatively neglected and remains primarily as a research tool. The reasons for this seem to be multiple. First, the complexity of preparing tissue samples for electron microscopy may have discouraged pathologists from attempting such an effort routinely. Although it is correct that tissues should be handled with care for good ultrastructural conservation, some reports show satisfactory conservation of ultrastructure in tissues fixed in $10 \%$ buffered formalin, which is also the routine fixation technique for optical microscopy. Second, the opinion that electron microscopy is not useful or essential for the diagnosis of neoplasias arose from the belief that there are no reliable ultrastructural criteria for the diagnosis of various neoplasias $[139,140]$.

In the 1980s, the emergence of poly and monoclonal antibodies brought solutions to almost every type of differential diagnosis in oncologic pathology. The overwhelming growth in the development of these antibodies has provided a very valuable tool for diagnosis $[141,142]$. The availability of a monoclonal antibody panel to evaluate function, structure, differentiation, and histogenesis at the morphological level can help overcome diagnostic problems while advancing our understanding and appreciation of neoplasias. Imonohistochemistry is a useful tool, easy to perform and of great specificity [143,111]. It is important to note that many monoclonal antibodies used in mammals and humans are useful for the immunomarking of fish cells and tissues [144,145].

\section{CONCLUSION}

Neoplasias in fish, unlike other diseases, do not generate great losses to aquaculture. However, the true value of neoplastic pathology compared is to better understand the histiogenesis and biological behavior of neoplasias in mammals and humans. Carcinogenesis is generally complex and in most neoplasias in both mammals and fish, the origin is unknown, and it seems that there are many factors that contribute to the onset and growth of neoplasias.

\section{ACKNOWLEDGEMENT}

This study was supported by the research funds from MCT/CNPq- Project \#301245/2016-09 $\mathrm{MCT} / \mathrm{CNPq} / \mathrm{CT}$ - Agronegocio/MPA Public Notice 036/2009 Project \#308013/2009-3, Coordenação de Aperfeiçoamento de Pessoal de Nível Superior (CAPES), and Ministério da Pesca e Aquicultura (MPA).

\section{COMPETING INTERESTS}

Authors have declared that no competing interests exist.

\section{REFERENCES}

1. Hajdu SI. A note from history: andmarks in history of cancer, part 1 . Cancer.

2011;117(5):1097-102.

DOI: $10.1002 /$ cncr.25553

Epub 2010 Oct 19.

PMID: 20960499.

2. Hajdu SI. A note from history: landmarks in history of cancer, part 2. Cancer. 2011;117(12):2811-20.

DOI: $10.1002 /$ cncr.25825

Epub 2010 Dec 29.

PMID: 21656759.

3. Willis RA. The spread of tumors in the human body. London: Butterworth \& co; 1952.

4. Muenst $S$, Läubli $H$, Soysal SD, Zippelius A, Tzankov A, Hoeller S. The immune system and cancer evasion strategies: Therapeutic concepts. J Intern Med. 2016;279(6):541-62.

DOI: $10.1111 /$ joim.12470

Epub 2016 Jan 8.

PMID: 26748421. 
5. Abbott M, Ustoyev $\mathrm{Y}$. Cancer and the immune system: The history and background of immunotherapy. Semin Oncol Nurs. 2019;35(5):150923.

DOI:10.1016/j.soncn.2019.08.002

Epub 2019 Sep 13.

PMID: 31526550.

6. Axelrod ML, Cook RS, Johnson DB, Balko JM. Biological consequences of MHC-II expression by tumor cells in cancer. Clin Cancer Res. 2019;25(8):2392-2402.

DOI: 10.1158/1078-0432.CCR-18-3200

Epub 2018 Nov 21.

PMID: 30463850.

PMCID: PMC6467754.

7. Damjanov I, Linder J. Neoplasia in Anderson's Pathology. 10th ed. Published by CRC Press.1995;640- 690.

ISBN 10: 0801672368.

8. Baranyi M, Lippai M, Szatmári Z. A stroma szerepe a tumorok kialakulásában és progressziójában [Role of the stroma in the initiation and progression of tumors]. Orv Hetil. Hungarian. 2015;156(45):1816-23.

DOI: 10.1556/650.2015.30294

PMID: 26522855.

9. Namba M, lijima M, Kondo T, Jahan I, Mihara K. Immortalization of normal human cells is a multistep process and a rate limiting step of neoplastic transformation of the cells. Hum Cell. 1993;6(4):253-9.

PMID: 8148305.

10. Winquist RJ, Hall $A B$, Eustace BK, Furey BF. Evaluating the immortal strand hypothesis in cancer stem cells: Symmetric/self-renewal as the relevant surrogate marker of tumorigenicity. Biochem Pharmacol. 2014;91(2):129-34.

DOI: 10.1016/j.bcp.2014.06.007

Epub 2014 Jun 24.

PMID: 24971715.

11. Harshbarger JC. Activities report of the registry of tumors in lower animals, 1975 supplement. Washington, DC: Smithsonian Institution; 1976.

12. Effron M, Griner L, Benirschke K. Nature and rate of neoplasia found in captive wild mammals, birds and reptiles at necropsy. J Natl Cancer Inst. 1977;59(1):185-98.

13. Hernandez-Divers SM, Garner MM. Neoplasia of reptiles with an emphasis on lizards. Vet Clin North Am Exot Anim Pract. 2003;6(1):251-73.

DOI: $10.1016 / \mathrm{s} 1094-9194(02) 00028-2$

PMID: 12616843.

14. Newton AL, Lewbart GA. Invertebrate oncology: Diseases, diagnostics, and treatment. Vet Clin North Am Exot Anim Pract. 2017;20(1):1-19.

DOI: 10.1016/j.cvex.2016.07.001

PMID: 27890285.

15. Ullrich $\mathrm{Cl}$, Aloni R, Saeed MEM, Ullrich W, Efferth T. Comparison between tumors in plants and human beings: Mechanisms of tumor development and therapy with secondary plant metabolites. Phytomedicine. 2019;64:153081.

DOI: 10.1016/j.phymed.2019.153081

Epub 2019 Sep 3.

PMID: 31568956.

16. Hason M, Bartunek P. Zebrafish models of cancer-new insights on modeling human cancer in a non-mammalian vertebrate. genes (Basel). 2019;10(11):935.

DOI: 10.3390/genes10110935.

PMID: 31731811.

PMCID: PMC6896156.

17. Mawdesley- Thomas LE. Neoplasia in fish. In: Ribelin LWE, Migaki G, editors. The pathology of fishes. USA: The University of Wisconsin Press. 1975;805-870. ISBN: 0-299-06520-0.

18. Reichenbach-Klinke $\mathrm{HH}$, Ahne W, Negele B, Ollenschlager W, Popp W, Spieser OH, Wolf K. Krankheiten und Schädigungen, 2th.ed. Gustav Fischer Verlag. Stuttgart, New York. 1980;507. ISBN: 84-200-0509-6.

19. Kinkelin P, Michel C, Ghittino P. Précis de pathologie des poissons. Editorial: Institut National de la Recherche. Paris: Paris Office International des Epizooties. 1985; 353.

ISBN: 84-200-0686-6.

20. Ferguson HW. Systemic pathology of fish. A text and atlas of comparative tissue responses in diseases of teleosts. Ames, IA: lowa State University Press. 1989;263. ISBN: 0813801478.

21. Stoskopf MK. Goldfish, koi, and carp neoplasia. In: Stoskopf MK, editor. Fish Medicine. London: W.B Saunders Company. 1993;490-491.

22. Carnevia D. Enfermedades de los peces ornamentales. Buenos aires argentina: Editorial agrovet. SA. 1993;319. ISBN: 950-9763-09-8.

23. Leatherland JF, Woo PTK. Fish diseases and disorders. Nosworthy Way Wallingford Oxfordshire, UK: CABI Head Office. 2010;2:403.

24. Roberts RJ. Neoplasia of teleosts. In: Roberts RJ, editor. Fish Pathology. 4th ed. 
Oxford: Blackwell Publishing Ltd. 2012;167-185.

25. Kalluri R. The biology and function of fibroblasts in cancer. Nat Rev Cancer. 2016;16(9):582-98.

DOI: $10.1038 /$ nrc. 2016.73

PMID: 27550820.

26. Lyssiotis CA, Kimmelman AC. Metabolic interactions in the tumor microenvironment. Trends Cell Biol. 2017;27(11):863-875.

DOI: $10.1016 /$ j.tcb.2017.06.003

Epub 2017 Jul 19.

PMID: 28734735

PMCID: PMC5814137.

27. Hinshaw DC, Shevde LA. The tumor microenvironment innately modulates cancer progression. Cancer Res. 2019;79(18):4557-4566.

DOI: $10.1158 / 0008-5472$.

CAN-18-3962.

28. Baba Al, Câtoi C. Comparative oncology. Bucharest (RO): The Publishing House of the Romanian Academy; 2007.

ISBN-10: 973-27-1457-3

29. Murphy B, Imai DM. Cutaneous Ossifying Fibroma in a Neon Tetra (Paracheirodon innesi). J Comp Pathol. 2016;155(23):272-275.

DOI:10.1016/j.jcpa.2016.06.007

Epub 2016 Jul 27.

PMID: 27476108.

30. Pilleri G. Uber Knochentumoren (Osteoma durum) bei Pogonias cromis Cuvier (Perciformes, Sciaenidae) von Los Cerros, Uruguay [Bone neoplasms (osteoma durum) in Pogonias cromis Cuvier (Perciformes, Sciaenidae) at Los Cerros, Uruguay]. Rev Suisse Zool, German. 1973;80(2):571-2.

PMID: 4748110.

31. Mesbah M, Rezaie A, Tulaby Dezfuly Z. Case report of chondroma in a grass carp (Ctenopharyngodon idella). Vet Res Forum. 2016;7(2):173-6.

Epub 2016 Jun 15.

PMID: 27482364.

PMCID: PMC4959347.

32. Singaravel V, Gopalakrishnan A, Raja K, Rajkumar M, Ferguson HW. Neoplasia in goldlined seabream, Rhabdosargus sarba (Forsskal, 1775). J Fish Dis. 2017;1-7. DOI: $10.1111 /$ jfd.12737

33. Macintyre PA. Tumors of the thyroid gland in teleost fishes. Zool Sci Contrib N Y Zool Soc. 1960;45:161-70.

PMID: 13764863.
34. Lombardini ED, Hard GC, Harshbarger JC. Neoplasms of the urinary tract in fish. Vet Pathol. 2014;51(5):1000-12.

DOI: $10.1177 / 0300985813511122$

Epub 2013 Dec 6.

PMID: 24318975.

35. Lu H, Zhu G, Fan L, Zhang L. Etiology and pathology of epidermal papillomas in allogynogenetic crucian carp Carassius auratus gibelio (female) x Cyprinus carpio var. singuonensis (male). Dis Aquat Organ. 2009;83(1):77-84.

DOI:10.3354/dao01985

PMID: 19301639.

36. Thiyagarajah A, Anderson MB, Hartley WR. Gonadal cysts in spotted gar (Lepisosteus oculatus) from Bayou Trepagnier, Louisiana, USA. Mar Environ Res. 2000;50(1-5):279-82.

DOI: $10.1016 / \mathrm{s} 0141-1136(00) 00049-0$ PMID: 11460704.

37. Bignold L. Principles of tumors. 1th ed. academic press. 2015;468. ISBN: 9780128015650.

38. Bowser PR, Casey JW, Casey RN, Quackenbush SL, Lofton L, Coll JA, Cipriano RC. Swimbladder Leiomyosarcoma in Atlantic Salmon (Salmo salar) in North America. Journal of Wildlife Diseases. 2012;48(3):795-798.

39. Sharon G, Benharroch D, Kachko L, ReisHevlin N, Zilberg D. Liposarcoma in clownfish, Amphiprion ocellaris Cuvier, produced in indoor aquaculture. J Fish Dis. 2015;38(6):575-80.

DOI: $10.1111 /$ jfd.12269

Epub 2014 Jun 11.

PMID: 24917512.

40. Dennis MM, Diggles BK. Multicentric orocutaneous fibrosarcoma in a fork-tailed catfish (Hemiarius dioctes). Journal of Fish Diseases. 2015;38:1077-1081.

DOI:10.1111/jfd.12349

41. Wicki G, Candarte P, Galli OM, Sal F, Luchini L, Romano LA. Alveolar rhabdomyosarcoma in the Carassius auratus (L.): Optical, immunohistochemical and ultrastructural study. Bulletin of the European Association of Fish Pathologists. 2016;36:237-245.

42. Ashley LM, Halver JE, Wellings SR. Case reports of three teleost neoplasms. Natl Cancer Inst Monogr. 1969;31:157-65. PMID: 4315898.

43. Baba Al. Neoplasm's classification in animals. Rom. Rev. Comp. One. 1999;1:36-44. 
44. Eloy C, Ferreira L, Salgado C, Soares P, Sobrinho-Simões M. Poorly Differentiated and Undifferentiated Thyroid Carcinomas. Turk Patoloji Derg. 2015;31(Suppl1):48-59. DOI: $10.5146 /$ tjpath.2015.01314

PMID: 26177317.

45. Romano LA, Tabata YA, Alexandrino AC. Histopathological and immunohistochemical diagnostic of carcinosarcoma of Mandible in a Rainbowtrout (Onchorhyincus mykiss). Acta Scientiae Veterinariae (UFRGS. Impresso). 2010;38:319-322.

46. Hisaoka KK. Congenital teratomata in the guppy, Lebistes reticulatus. J Morphol. 1961;109:93-113.

DOI: 10.1002/jmor.1051090107

PMID: 13907766.

47. Newman SJ, Brown CJ, Patnaik AK. Malignant ovarian teratoma in a red-eared slider (Trachemys scripta elegans). Journal of Veterinary Diagnostic Investigation. 2003;15:77-81.

48. Romanucci $M$, Arbuatti A, Massimini M, Defourny SVP, Della Salda L. Ovarian teratoma in an adult female Zoogoneticus tequila (Webb \& Miller 1998): Histological and immunohistochemical features. J Fish Dis. 2017;40(6):859-862.

DOI: $10.1111 /$ jfd. 12553

Epub 2016 Sep 5.

PMID: 27592861.

49. Ok CY, Woda B, Kurian E. Pathology of cancer. In: Pieters RS, Liebmann J, eds. Cancer Concepts: A Guidebook for the Non- Oncologist. Worcester, MA: University of Massachuset's Medical School; 2018.

DOI: 10.7191/cancer concepts.1023

50. Klosterhoff MC, Rodrigues RV, Sampaio LA, Pedrosa VF, Haas E, Luchini L, Wicki $\mathrm{G}$, Romano LA. Occurrence of a renal lymphoma Type B in cobia, Rachycentron canadum (L). Bulletin of the European Association of Fish Pathologists. 2019;39:72-77.

51. Whyte RI, Donington JS. Hamartomas of the lung. Semin Thorac Cardiovasc Surg. 2003;15(3):301-4.

DOI: $10.1016 / \mathrm{s} 1043-0679(03) 70010-0$

PMID: 12973708.

52. Vorbach BS, Wolf JC, Yanong RP. Odontomas in two long-finned ocellaris clownfish (Amphiprion ocellaris). J Vet Diagn Invest. 2018;30(1):136-139. DOI: $10.1177 / 1040638717729726$ Epub 2017 Sep 14.
PMID: 28906183.

PMCID: PMC6504150.

53. Litjens $\mathrm{G}$, Sánchez $\mathrm{Cl}$, Timofeeva $\mathrm{N}$, Hermsen M, Nagtegaal I, Kovacs I, Hulsbergen-van de Kaa $C$, Bult $P$, van Ginneken B, van der Laak J. Deep learning as a tool for increased accuracy and efficiency of histopathological diagnosis. Sci Rep. 2016;6:26286.

DOI: $10.1038 /$ srep26286

PMID: 27212078.

PMCID: PMC4876324.

54. MacEwen GE, Khanna C, Radinsky R. Cancer biology and metastasis. In: Withrow SJ, MacEwen GE, editors. Small animal clinical oncology. 3th ed. Philadephia: W.B. Saunders. 2001;18-34.

55. Carr NJ, Bibeau F, Bradley RF, Dartigues $P$, Feakins RM, Geisinger KR, Gui X, Isaac $\mathrm{S}$, Milione M, Misdraji J, Pai RK, Rodriguez-Justo M, Sobin LH, van Velthuysen MF, Yantiss RK. The histopathological classification, diagnosis and differential diagnosis of mucinous appendiceal neoplasms, appendiceal adenocarcinomas and pseudomyxoma peritonei. Histopathology. 2017;71(6):847858.

DOI: $10.1111 /$ his.13324

Epub 2017 Sep 19

PMID: 28746986.

56. Vilalta L, Meléndez-Lazo A, Doria G, Ramis A, Solano-Gallego L, Pastor J, Martorell J. Clinical, cytological, histological and immunohistochemical features of cutaneous mast cell tumours in ferrets (Mustela putorius furo). J Comp Pathol. 2016;155(4):346-355.

DOI: 10.1016/j.jcpa.2016.07.012

Epub 2016 Aug 28

PMID: 27578524.

57. Sirri R, Diana A, Scarpa F, Brachelente C, Vitellozzi G, Ceredi L, Mandrioli L. Ultrasonographic and pathologic study of schwannoma in a Goldfish (Carassius auratus). Vet Clin Pathol. 2015;44(4):58691.

DOI: $10.1111 / \mathrm{vcp} .12285$

Epub 2015 Sep 10.

PMID: 26356489.

58. Steinbeck RG. Pathologic mitoses and pathology of mitosis in tumorigenesis. Eur J Histochem. 2001;45(4):311-8.

DOI: $10.4081 / 1640$

PMID: 11845999. 
59. Therman E, Kuhn EM. Mitotic modifications and aberrations in cancer. Crit Rev Oncog. 1989;1(3):293-305 PMID: 2488134.

60. Amend SR, Torga G, Lin KC, Kostecka LG, de Marzo A, Austin RH, Pienta KJ. Polyploid giant cancer cells: Unrecognized actuators of tumorigenesis, metastasis, and resistance. Prostate. 2019;79(13):1489-1497.

DOI: $10.1002 /$ pros. 23877

Epub 2019 Aug 2.

PMID: 31376205.

PMCID: PMC6706309.

61. Smolarz K, Renault T, Wolowicz M. Ultrastructural study of neoplastic cells in Macoma balthica (Bivalvia) from the Gulf of Gdansk (Poland). J Invertebr Pathol. 2006 Jun;92(2):79-84.

DOI: $10.1016 /$ j.jip.2006.04.003

PMID: 16723136.

62. Fukumura D, Jain RK. Tumor microvasculature and microenvironment: targets for anti-angiogenesis and normalization. Microvasc Res. 2007 SepNov;74(2-3):72-84.

DOI: 10.1016/j.mvr.2007.05.003

Epub 2007 May 18.

PMID: 17560615.

PMCID: PMC2100036.

63. Klosterhoff $M$, Sampaio L, Okamoto $M$, Pedrosa V, Romano LA. Hemangioma esclerosante mandibular em Paralichthys orbignyanus: relato de caso. Arquivo Brasileiro de Medicina Veterinária e Zootecnia. 2018;70:793-796.

64. Romano LA, Klosterhoff $M$, Führ $F$, Rodrigues RV, Garrido-Pereira MA, Tesser MB, Sampaio LA. Borderline ovarian mucinous cystadenoma with invasion of stroma in the goldfish Carassius auratus (L.). Bulletin of The European Association of Fish Pathologists. 2014;34:25-31.

65. Miyazaki T, Asai Y, Kobayashi T, Miyata M. Lympholeukemia in madai Pagrus major in Japan. Diseases of Aquatic Organisms. 2000;40(2):147-55.

DOI:10.3354/dao040147

66. Chaffer CL, Weinberg RA. A perspective on cancer cell metastasis. Science. 2011;331(6024):1559-64.

DOI: $10.1126 /$ science.1203543

PMID: 21436443.

67. Chiang SP, Cabrera RM, Segall JE. Tumor cell intravasation. Am J Physiol Cell Physiol. 2016;311(1):C1-C14.

DOI: 10.1152/ajpcell.00238.2015
Epub 2016 Apr 13.

PMID: 27076614.

PMCID: PMC4967137.

68. Suhail $\mathrm{Y}$, Cain MP, Vanaja K, Kurywchak PA, Levchenko A, Kalluri R, Kshitiz. Systems biology of cancer metastasis. Cell Syst. 2019;9(2):109-127.

DOI: 10.1016/j.cels.2019.07.003

PMID: 31465728.

PMCID: PMC6716621.

69. Romano LA, Cuevas F. Producción Experimental de Melanomas Cutáneos en el Pez Espada Xiphophorus helleri (Heckel) y el Pez Platis, Xiphophorus Maculatus (Gunther) (Pisces Poecilidae). Revista de La Asociación de Ciencias Naturales Del Litoral. 1987;18:185-189.

70. Fernández AA, Bowser PR. Two cases of non-hybrid melanoma formation in Xiphophorus nezahualcoyotl Rauchenberger, Kallmann \& Morizot. Journal of Fish Biology. 2008;72:292-300.

71. Fischer $\mathrm{AH}$, Jacobson $\mathrm{KA}$, Rose J, Zeller R. Hematoxylin and eosin staining of tissue and cell sections. CSH Protoc. 2008; pdb.prot4986.

72. Narla LD, Newman B, Spottswood SS, Narla S, Kolli R. Inflammatory pseudotumor. Radiographics. 2003;23:719-29.

DOI: $10.1148 / \mathrm{rg} .233025073$

73. Romano LA, Pedrosa VF. Re-claiming H\&E: back to the future. Postgraduate Medical Journal. 2019;136955-2.

74. Romano LA, Marozzi AV. Epithelioreticular cell thymoma in carp Cyprinus carpio L: A case with ultrastructural study. Journal of Fish Diseases (Print). 2004;27:369-373.

75. Romano LA, Klosterhoff $M$, Führ $F$, Gusmão EP, Garrido-Pereira MA, Sampaio LA, Tesser MB. Neoplasia of the sertoli cells in wild carp, Cyprinus carpio: Optical, immunohistochemical and ultrastructural study. Bulletin of The European Association of Fish Pathologists. 2013;33:84.

76. Romano LA, Klosterhoff $M$, Führ $F$, Garrido-Pereira MAR, Pedrosa VF. Multiple neurofibromas of the heart 1 in wild carp, Cyprinus carpio: Optical, immunohistochemical and ultrastructural study. Bulletin of The European Association of Fish Pathologists. 2015;34:201-207.

77. Romano LA, Wicki G, Candarte P, Galli OM, Sal F, Luchini L, Klosterhoff $M$, 
Pedrosa VF. Melanoma in a Carassius auratus (L.): Study with optical microscopy, electron microscopy and immunohistochemistry. Bull. Eur. Ass. Fish Pathol. 2018;38(6):240-248.

78. Romano LA, Klosterhoff MC, Medeiros AFF, Pedrosa VF. Occurrence of spontaneous melanoma in swordtail fish (Xiphophorus hellerii). Study with optical microscopy, immunohistochemistry and electron microscopy. Bull. Eur. Ass. Fish Pathol. 2020;40(4):165-172.

79. Rezaie A, Alishahi M, Dezfuly ZT. The first report on epicardial cavernous hemangioma in farmed sturgeon, Acipenser baerii, a microscopic survey. Iranian Journal of Fisheries Sciences. 2018;17(4):835-839.

DOI: $10.22092 / I J F S .2018 .115482$

80. Rahmati-Holasoo $H$, Soltani $M$, Masoudifard M, Shokrpoor S, Mirghaed AT, Ahmadpoor M. Nephroblastoma in bester sturgeon, a cultured hybrid of Huso huso $\times$ Acipenser ruthenus: Diagnostic imaging, clinical and histopathological study. J Fish Dis. 2018;1-9.

81. Hayashi S, Furukawa S, Abe M, Usuda K, Ogawa I, Miyamoto Y. Lymphoma in a japanese killifish. J Toxicol Pathol. 2008;21:115-117.

Available:https://doi.org/10.1293/tox.21.11 5

82. Marino F, Lanteri G, Manganaro M, Macrì F. European Eel Stomatopapillomatosis in the south Tyrrhenian sea: surgical excision and post surgical recovery. Bull. Eur. Ass. Fish Pathol. 2010;30(1):30-34.

83. Gjurcevic E, Kužir S, Sfacteria A, Drašner K, Marino F. Spontaneous multicentric myxoma of the dermal nerve sheaths in farmed European eels Anguilla anguilla. Dis Aquat Org. 2014;111:173-176.

DOI: $10.3354 /$ dao02746

84. Caruso D, Keith P, Plassiart G. Skin nodular neoplasm in the armoured catfish, cascarudo (Callichthys callichthys), in French Guiana, South America. J. Appl. Ichthyol. 2003;19:126-128.

Available:https://doi.org/10.1046/j.14390426.2003.00447.x

85. Lin CC, Hung SW, Tu CY, Tsou LT, Shieh MT, Lin SL, Liu PC, Wang WS. Spontaneous stomach lymphoma and liver metastases in flower horn fish, hybrid cichlid. Bulletin of European Association of Fish Pathologists. 2008; 28:115-120.
86. Hochwartner O, Loupal G, Wildgoose WH, Schmidt-Posthaus $\mathrm{H}$. Occurrence of spontaneous tumours of the renal proximal tubules in oscars Astronotus ocellatus. Dis Aquat Org. 2010;89:185-189.

DOI: $10.3354 /$ dao02149

87. Rahmati-holasoo $H$, Hobbenaghi $R$, Tukmechi A Morvaridi A. The case report on squamous cell carcinoma in Oscar (Astronotus ocellatus). Comp. Clin. Pathol. 2010;19(4):421-424.

DOI 10.1007/s00580-010-0963-z

88. Rahmati-Holasoo $\mathrm{H}$, Shokrpoor $\mathrm{S}$, Tavakkoli A, Vajhi A, Mousavi HE. Liposarcoma or invasive lipomatosis in flower horn fish, hybrid cichlid: Clinical, radiological, ultrasonographical and histopathological study. Journal of Fish Diseases. 2016;39:309-315.

DOI:10.1111/jfd.12364

89. Okorie-Kanu CO, Agbakwuru IO, Nwagbara ND. Suspected Case of Rhabdomyosarcoma in a Cultured Clarias gariepinus. Nigerian Veterinary Journal. 2017;38(3).

90. Camus MS, Hyatt MW, Clauss TM, Berliner AL, Camus AC. Chromatophoroma in a crevice kelpfish (Gibbonsia montereyensis). Vet Clin Pathol. 2011;40(4):549-552.

DOI:10.1111/j.1939-165x.2011.00365.x

91. Ramos P, Peleteiro MC. Três casos de neoplasias espontâneas em peixes. RPCV. 2003;98 (546):77-80.

92. Marino $F$, Licata L, Albano $M$, Ieni $A$, Caro1 GD, Macrì B. Angioleiomyoma in a conger (Conger conger). Dis Aquat Org. 2016;119:85-89.

DOI: $10.3354 /$ dao02984

93. Quaglio F, Zappulli V, Poppi L, Capovilla $P$, Capparucci F, Marino F. Squamous cell carcinoma in a wild European bullhead Cottus gobio. Dis Aquat Org. 2016;122:7376. DOI: $10.3354 /$ dao03065

94. O'Hagan BJ, Raidal SR. Surgical removal of retrobulbar hemangioma in a goldfish (Carassius auratus). Vet Clin Exot Anim. 2006;9:729-733.

DOI: 10.1016/j.cvex.2006.05.028

95. Raidal SR, Shearer PL, Stephens F, Richardson J. Surgical removal of an ovarian tumour in a koi carp (Cyprinus carpio). Australian Veterinary Journal. 2006;84(5):178-181.

Available:https://doi.org/10.1111/j.17510813.2006.tb12776.x 
96. Knüsel $\mathrm{R}$, Brandes $\mathrm{K}$, Lechleiter $\mathrm{S}$, Schmidt-Posthaus $\mathrm{H}$. Two independent cases of spontaneously occurring branchioblastomas in koi carp (Cyprinus carpio). Vet. Pathol. 2007;44:237-239.

DOI: 10.1354/vp.44-2-237

97. Sholichah L, Lusiastuti AM, Caruso D, Subamia IW, Purwaningsih U. Tumor case in koi carp (Cyprinus carpio). Indonesian Aquaculture Journal. 2010;5(2):139-145. DOI:http://dx.doi.org/10.15578/iaj.5.2. 2010

98. Stegeman N, Heatley JJ, Rodrigues A, Pool R. Nephroblastoma in a koi (Cyprinus carpio). Journal of Exotic Pet Medicine. 2010;19(4):298-303.

Available:https://doi.org/10.1053/j.jepm.20 10.10.009

99. Sahoo PK, Mohanty BR, Das A, Mahapatra KD, Saha JN. Cavernous haemangioma in cultured olive barb, Puntius sarana (Hamilton). Journal of Fish Diseases. 2012;35:695-697.

DOI:10.1111/j.1365-2761.2012.01370.x

100. Marino F, Lanteri G, Rapisarda G, Perillo $A$, Macrì $B$. Spontaneous schwannoma in zebrafish, Danio rerio (Hamilton). Journal of Fish Diseases. 2012;35:239-242.

DOI: 10.1111/j.1365-2761.2011.01335.x

101. Hyatt MW, Clauss TM, Dennison SE, Camus AC. Retroperitoneal hemangiosarcoma in a common carp Cyprinus carpio: A case report. Dis Aquat Org. 2013;107:151-160.

DOI: $10.3354 /$ dao02675

102. Mandrioli L, Sirri R, Gustinelli A, Quaglio F, Sarli G, Chiocchetti R. Ocular glioneuroma with medulloepitheliomatous differentiation in a goldfish (Carassius auratus). Journal of Veterinary Diagnostic Investigation. 2014;26(1):167-172.

DOI: $10.1177 / 1040638713515218$

103. Oryan A, Alidadi S, Shayegh $H$, Geramizadeh B. Cutaneous Leiomyoma in a Goldfish Carassius auratus. Fish Pathology. 2015;50 (3):112-114.

104. Paquette CE, Kent ML, Peterson TS, Wang $\mathrm{R}$, Dashwood $\mathrm{RH}$, Lohr CV. Immunohistochemical characterization of intestinal neoplasia in zebrafish Danio rerio indicates epithelial origin. Diseases of Aquatic Organisms. 2015;116(3):191-197. https://doi.org/10.3354/dao02924

105. Lanteri G, Ieni A, Toffan A, Abbate J, Saraò $M$, Barresi $V$, Macrì $B$. Immunohistochemical patterns of a nonviral papilloma in Goldfish (Carassius auratus, L.). Bull. Eur. Ass. Fish Pathol. 2016;36(5):208-213.

106. Rezaie A, Mousavi SM, Ansari MB. Rhabdomyosarcoma in a Silver Carp. Journal of Aquatic Animal Health. 2016;28:118-121. Hilly M, Adams ML, Nelson SC. A study of digit fusion in the mouse embryo. Clin Exp Allergy. 2002;32(4):489-98.

107. Shokrpoor S, Sasani F, Rahmati-Holasoo $\mathrm{H}$, Zargar A. Concurrence of a fibroma ad myxoma in an oranda goldfish (Carassius auratus). Bull. Eur. Ass. Fish Pathol. 2016;36(6):263-268.

108. Sirri R, Pretto T, Montesi F, Berton V, Mandrioli L, Barbé T. Hikui disease in nine koi carp (Cyprinus carpio): First description of a cutaneous perivascular wall tumour. Vet Dermatol. 2016;27:301-e74.

DOI: $10.1111 /$ vde. 12338

109. Rezaie A, Dezfuly ZT, Peyghan R. Fibrosarcoma in a Goldfish (Carassius auratus): A Case report. IJVST. 2017a;9(1):45-48.

DOI: $10.22067 /$ veterinary.v9i1.58619

110. Siniard WC, Sheley MF, Stevens BN, Parker-Graham CA, Roy MA, Sinnott DM, Watson KD, Marinkovich MJ, Robertson JA, Frei S, Soto E. Immunohistochemical analysis of pigment cell tumors in two cyprinid species. Journal of Veterinary Diagnostic Investigation. 2019;31(5):788791.

DOI: $10.1177 / 1040638719864380$

111. Iaria $C$, leni $A$, Corti I, Puleio R, Brachelente C, Mazzullo G, Lanteri G. Immunohistochemical study of four fish tumors. J Aquat Anim Health. 2019;31(1):97-106.

DOI: 10.1002/aah.10058

Epub 2019 Feb 5.

PMID: 30554413.

112. Williams Jr EH, Rand TG, BunkleyWilliams L. Neurofibromas in gray snappers, Lutjanus griseus, from bermuda and the unusual distribution of nerve sheath tumors in snappers at the northern extremes of West Indian Waters. Caribbean Journal of Science. 2000;36(34):344-346.

113. Marino F, Chiofalo B, Mazzullo G, Panebianco A. Multicentric infiltrative lipoma in a farmed Mediterranean seabass Dicentrarchus labrax: A pathological and biochemical case study. Dis Aquat Organ. 2011;6;96(3):259-64.

DOI: $10.3354 /$ dao02378 
114. Marino F, Macrì D, Lanteri G, Manganaro M, Monaco S, Germanà A. Neurofibroma in a striped mullet: Histochemical and immunohistochemical study. Journal of Aquatic Animal Health. 2010;22:92-94.

115. Iwanowicz LR, Goodwin AE, Harshbarger JC. Embryonal rhabdomyosarcoma of the giant gourami, Colisa fasciata (Bloch \& Schneider). Journal of Fish Diseases. 2001;24:177-179.

DOI: $10.1046 / \mathrm{j} .1365-2761.2001 .00290 . x$

116. Lombardini ED, Law M, Lewis BS. Nephroblastoma in two siamese fighting fish Betta splendens. Fish Pathology. 2010;45(3):137-139.

DOI: $10.3147 /$ jsfp. 45.137

117. Rahmati-Holasoo $\mathrm{H}$, Alishahi $\mathrm{M}$, Shokrpoor S, Jangarannejad A, Mohammadian B. Invasion of melanoma to angiolipoma in a male Siamese fighting fish, Betta splendens, Regan. Journal of Fish Diseases. 2015;38(10):925-930. DOI:10.1111/jfd.12301

118. Rahmati-Holasoo H, Pedram MS, Mousavi HAE, Shokrpoor S, Lewbart GA. Malignant iridophoroma in a male Siamese fighting fish (Betta splendens) Regan: A clinical, surgical and histopathological study. Bull. Eur. Ass. Fish Pathol. 2019;39(3):106-113.

119. Silva EG da, Gionfriddo JR, Powell CC, Campbell TW, Ehrhart EJ. Iridociliary melanoma with secondary lens luxation: Distinctive findings in a long-horned cowfish (Lactoria cornuta). Veterinary Ophthalmology. 2010;13(1):123-127.

Available:https://doi.org/10.1111/j.14635224.2010.00804.x

120. Bowser PR, Abou-Madi N, Garner MM, Bartlett SL, Grimmett SG, Wooster GA, Paul TA, Casey RN, Casey JW. Fibrosarcoma in yellow perch, Perca flavescens (Mitchill). Journal of Fish Diseases. 2005;28:301-305.

Available:https://doi.org/10.1111/j.13652761.2005.00620.x

121. Stefano C de, Bonfiglio R, Montalbano G, Giorgianni P, Lanteri G. Multicentric lipoma in a molly (Poecilia velifera). Bulletin European Association of Fish Pathologists. 2012;32(6):220-224.

122. Rahmati-holasoo $\mathrm{H}$, Shokrpoor $\mathrm{S}$, Enayati A, Zargar A, Ahmadpoor M. Congenital teratoma in guppy (Poecilia reticulata) in Iran. Poeciliid Research. 2014;4(1):1-5. Available:http://www.pr.bioflux.com.ro/

123. Rezaie A, Dezfuly ZT, Peyghan R. Occurrence of squamous cell carcinoma in a platy fish (Xiphophorus maculatus): A case report. Iranian Journal of Fisheries Sciences. 2017;16(4):1349-1353.

124. Lumsden JS, Marshall S. Sporadic neoplasms of farmed chinook salmon, Oncorhynchus tshawytscha (Walbaum), from New Zealand. Journal of Fish Diseases. 2003;26:393-399.

DOI: 10.1046/j.1365-2761.2003.00474.x

125. Sirri R, Bianco C, Beraldo P, Mandrioli L, Pulvirenti I, Brachelente C, Galeotti M, Sarli G. Rhabdomyosarcoma of soft tissues in an adult brook trout (Salvelinus fontinalis). J. Comp. Path. 2015;153:190195.

Available:http://dx.doi.org/10.1016/j.jcpa.2 015.05.001

126. Soares F, Leitão A, Moreira M, Sousa JT de, Almeida AC, Barata M, Feist SW, Pousão-Ferreira P, Ribeiro L. Sarcoma in the thymus of juvenile meagre Argyrosomus regius reared in an intensive system. Dis Aquat Org. 2012;102:119-127. DOI: 10.3354/dao02545

127. Marino $F$, Monaco $S$, Salvaggio $A$, Macrì B. Lipoma in a farmed northern bluefin tuna, Thunnus thynnus (L.). Journal of Fish Diseases. 2006;29:697-699.

DOI: 10.1111/j.1365-2761.2006.00764.x

128. Johnston CJ, Deveney MR, Bayly T, Nowak BF. Gross and histopathological characteristics of two lipomas and a neurofibrosarcoma detected in aquacultured southern bluefin tuna, Thunnus maccoyii (Castelnau), in South Australia. Journal of Fish Diseases. 2008;31:241-247.

DOI: 10.1111/j.1365-2761.2007.00863.x

129. Weisse C, Weber ES, Matzkin Z, Klide A. Surgical removal of a seminoma from a black sea bass. JAVMA. 2002; 221(2).

DOI: 10.2460/javma.2002.221.280

130. Sweet M, Kirkham N, Bendall M, Currey L, Bythell J, Heupel M. Evidence of melanoma in wild marine fish populations. PLoS ONE. 2012;7(8):e41989.

Available:https://doi.org/10.1371/journal.po ne.0041989

131. Marino F, Germanà A, Panebianco A. A case of schwannoma in farmed seabream Sparus aurata. Dis Aquat Org. 2008;82:249-252.

DOI: $10.3354 /$ dao01992

132. Gómez S. Multiple dermal lipomas in farmed striped seabream Lithognathus 
mormyruson the Spanish Mediterranean coast. Dis Aquat Org. 2009;85:77-79.

DOI: $10.3354 /$ dao02062

133. Singaravel V, Gopalakrishnan A, Raja K, Vijayakumar R. Leiomyosarcoma in pickhandle barracuda, Sphyraena jello Cuvier, 1829. J Appl Ichthyol. 2017;33:807-809.

Available:https://doi.org/10.1111/jai.13366

134. Willens S, Dunn JL, Frasca Jr S. Fibrosarcoma of the brood pouch in an aquarium-reared lined seahorse (Hippocampus erectus). Journal of Zoo and Wildlife Medicine. 2004;35(1):107-109. DOI: $10.1638 / 02-085$

135. LePage V, Dutton, Christopher J, Kummrow M, McLelland DJ, Young K, Lumsden JS. Neoplasia of captive yellow sea horses (Hippocampus kuda) and weedy sea dragons (Phyllopteryx taeniolatus). Journal of Zoo and Wildlife Medicine. 2012;43(1):50-58.

Available:https://doi.org/10.1638/20100236.1

136. Stilwell JM, Boylan SM, Howard S, Camus AC. Gas gland adenoma in a lined seahorse, Hippocampus erectus, Perry 1810. Journal of Fish Diseases. 2018;41(1):171-174.

Available:https://doi.org/10.1111/jfd.12677

137. Lima FC, Souza APM, Mesquita EFM, Souza GN, Chinelli VCJ. Osteomas in cutlass fish, Trichiurus lepturus L, from Niteroi, Rio de Janeiro state, Brazil. J. Fish. Dis. 2002;25(1):57-61.

Available:https://doi.org/10.1046/j.13652761.2002.00336.x

138. Gurcan MN, Boucheron LE, Can A, Madabhushi A, Rajpoot NM, Yener B.
Histopathological image analysis: a review. IEEE Rev Biomed Eng. 2009;2:147-171. DOI:10.1109/RBME.2009.2034865

139. Mackay B, Osborne BM. The contribution of electron microscopy to the diagnosis of tumors. Pathobiol Annu. 1978;8:359-405. PMID: 214739.

140. Eyden B. Electron microscopy in tumour diagnosis: Continuing to complement other diagnostic techniques. Histopathology. 1999;35:102-108.

DOI: 10.1046/j.1365-2559.1999.0741b.x

141. Tan HT, Low J, Lim SG, Chung MC. Serum autoantibodies as biomarkers for early cancer detection. FEBS J. 2009;276:6880-6904.

DOI: $10.1111 / \mathrm{j} .1742-4658.2009 .07396 . x$

142. Zhang X, Soori G, Dobleman TJ, Xiao GG. The application of monoclonal antibodies in cancer diagnosis. Expert Rev Mol Diagn. 2014;14:97-106.

DOI: $10.1586 / 14737159.2014 .866039$

143. Zola H, Roberts TP. Monoclonal antibodies: diagnostic uses. In: Encyclopedia of Life Sciences (ELS). John Wiley \& Sons, Ltd: Chichester, NJ, USA; 2001.

DOI:10.1002/9780470015902.a0001205.p ub3

144. Romano LA, Marozzi V, Zenobi C.Utilizacion de anticuerpos humanos en la marcación de receptores CD3 y CD4 de linfocitos en Xiphophorus hellerii. RevSoc Científica Argent. 2004;43:123-127.

145. Batista RC, Figueiredo MA, Almeida DV, Romano LA, Marins LF. Impairment of the immune system in $\mathrm{GH}$-overexpressing transgenic zebra fish (Danio rerio). Fish Shellfish Imm. 2014;36:519-524.

(c) 2020 Romano and Pedrosa; This is an Open Access article distributed under the terms of the Creative Commons Attribution License (http://creativecommons.org/licenses/by/4.0), which permits unrestricted use, distribution, and reproduction in any medium, provided the original work is properly cited.

Peer-review history:

The peer review history for this paper can be accessed here: http://www.sdiarticle4.com/review-history/64379 